\title{
Development and Evaluation of Solbrax-Water Nanoemulsions for Removal of Oil from Sand
}

\author{
Priscila F. Oliveira, Thiago M. Oliveira, Luciana S. Spinelli, and Claudia R. E. Mansur \\ Institute of Macromolecules, Federal University of Rio de Janeiro, Avenue Horácio Macedo, 2030, Ilha do Fundão, \\ 21941-598 Rio de Janeiro, RJ, Brazil \\ Correspondence should be addressed to Luciana S. Spinelli; spinelli@ima.ufrj.br
}

Received 8 January 2014; Accepted 26 February 2014; Published 30 March 2014

Academic Editor: Jie-Fang Zhu

Copyright (c) 2014 Priscila F. Oliveira et al. This is an open access article distributed under the Creative Commons Attribution License, which permits unrestricted use, distribution, and reproduction in any medium, provided the original work is properly cited.

In recent years, surfactants have been used to clean up soils and aquifers contaminated by petroleum and petroleum derivatives. The purpose of this study was to develop and evaluate nanoemulsions for remediation of soil contaminated by petroleum, by using a commercial solvent Solbrax. The nanoemulsions were prepared by the phase inversion temperature (PIT) method, using oil phase Solbrax (a solvent extracted from naphtha with low aromatics content) and a nonionic ethoxylated lauryl ether surfactant. The surfactant concentrations were varied from 10 to $12 \mathrm{wt} \%$ and the oil phase was varied from 5 to $20 \mathrm{wt} \%$. A $2^{3}$ factorial experimental design with center point run was used to evaluate the soil washing process, varying time, temperature, and shear rate of the system. The results show that the most efficient system (with $90 \%$ efficiency) was that using the nanoemulsion containing $5 \mathrm{wt} \%$ of Solbrax and $12 \mathrm{wt} \%$ of surfactant after four hours of washing, on 240 rotation $\cdot \mathrm{min}^{-1}$ of shear rate and at a temperature of $318 \mathrm{~K}$.

\section{Introduction}

Contamination of the soil, water, and air has increased greatly in recent years. One of the main contributors is the petroleum industry, which causes contamination through release of various organic compounds along its productive chain. Among the contamination sources are fuel leaks during transport and spills caused by traffic accidents $[1,2]$.

Various chemical and physical treatment processes have been developed and tested to remove hydrocarbons from the soil. The processes used to contain spills on land vary according to the quantity and type of oil or derivative and soil. For example, the less viscous the oil is and the more porous the soil is, the faster the spill will spread [3-5].

Under natural conditions, Kuyukina et al. have shown that oil's penetration in the soil is a complex process that involves many chemical, physical, and biological factors. Crude oil is highly hydrophobic, as most of its components, with very low solubility in water [6]. According to the oil's origin, its chemical composition and physical properties can vary greatly, affecting the methods of treating contaminated areas. Physical characteristics of the oil such as density, temperature and surface/interfacial tension all affect the mechanisms that can be used to remove oil from soil without causing chemical destruction or modification [7].

Chemical products such as surfactants have been used to remediate soils and aquifers contaminated with petroleum derivatives. Surfactants are compounds that have affinity for both water and oil, because their molecules (called amphiphilic molecules) have two functional groups: one that is hydrophilic and one that is lipophilic. This structural arrangement creates a monolayer at the water-oil interface, reducing the interfacial tension and the mobility of the contaminant $[3,7]$.

Since most organic contaminants are hydrophobic, the objective of using surfactants is to reduce the interfacial tension organic contaminant/water to increase its separation from the soil, both from the surface and pores of the grains. Under certain circumstances, electrolytes can also be used in aqueous surfactant solutions, with the aim of increasing the surfactant's efficiency in removing the oil $[6,8]$.

Although the use of surfactants to remediate soil contaminated by hydrocarbons has excellent potential, one of the drawbacks of this technique is the formation of macroemulsions, which are thermodynamically unstable and can break 
down and form two immiscible phases consisting of oil and water. Besides this, due to the relatively large size of the droplets in the macroemulsion (between 100 and $1000 \mathrm{~nm}$ in diameter), these droplets tend to obstruct the pores during their transport in the soil, hindering the clean-up process $[3,9]$.

Oliveira and Maldonado [9] show that the use of microemulsions as an alternative to clean up areas contaminated by heavy oil fractions has been shown to have great potential. Among the advantages are these molecules' high capacities to dissolve the oil phase in aqueous systems and their good efficiency in remediation at ambient temperature. Vegetable oils and vegetable oils based nanoemulsions have shown one sustainable alternative for removal of oil of contaminated systems $[10,11]$.

Microemulsions and nanoemulsions are similar systems composed of a phase containing droplets of nanometric size (10 to $200 \mathrm{~nm}$ ) dispersed in a continuous liquid medium. The main differences between these two systems, when both are prepared using surfactants, are related to the concentration of the surfactants used (nanoemulsions are prepared in the presence of lower surfactant concentrations in comparison with microemulsion), to the presence of cosurfactants (nanoemulsions do not require the presence of cosurfactants) and the methods used to prepare them [12-17].

Nanoemulsions can be prepared with high- and low-energy emulsification. In low-energy emulsification, nanoemulsions are prepared by altering the physicalchemical properties of the system. This method uses the energy stored within the molecular aggregates formed by the surfactant molecules present in the emulsion [18]. Among the low-energy emulsification methods is induced transitional phase inversion, also known as the phase inversion temperature (PIT) method, which uses a particular characteristic of emulsions stabilized by ethoxylated nonionic surfactants. In a fixed composition of the emulsion, the relative affinity of the surfactant for the different phases is altered and controlled by the temperature [19]. This phenomenon is due to the specific solubility of nonionic surfactants in function of temperature, reflected in the behavior of the phases of an emulsion [20].

In this work we prepared oil/water nanoemulsions using Solbrax Eco 225/255 and nonionic ethoxylated lauryl ether surfactant, applying the PIT method and characterizing them regarding their stability and particle size. We then evaluated these systems, according to the efficiency in removing the oil on sand contaminated with petroleum, by the variation of temperature, time, and shear rate washing with nanoemulsion flushing.

\section{Experimental Procedure}

2.1. Determination of the Cloud Point of the Nonionic Surfactants. For using low energy emulsification, it is necessary to identify the surfactants solubilization properties that change. We used the nonionic ethoxylated lauryl ether surfactant Ultrol L70, obtained from Oxiteno, Brazil (Ultrol L line), with seven ethylene oxide (EO) units in its chains. As the aqueous phase, we used distilled deionized water.
The cloud points of the aqueous surfactant solutions are the main measure to view this properties alteration. They were measured visually, using a test tube containing the surfactant solution immersed in a beaker with water heated over a hotplate. The temperature range analyzed was from 293 to $343 \mathrm{~K}$, measured by a thermometer placed in the solution. The cloud point was determined by the average between the temperature at which the first visual indication of clouding occurred and the temperature at which it disappeared. In these analyses, aqueous solutions of Ultrol L70 were used at concentrations of 10,11 , and $12 \mathrm{wt} \%$.

2.2. Measurement of the Oil/Water Interfacial Tension. The interfacial tension of the oil phase with the aqueous surfactant solution (Ultrol L70) was measured to observe the behavior of the surfactant at the water-oil interface. $40 \mathrm{~g}$ of aqueous surfactant solutions was prepared, at the same above concentrations. They were then left at rest for 24 hours to allow the system to dissolve completely. The interfacial tension measurements were performed with a Krüss K10 digital tensiometer, which uses the du Noüy ring method.

As the oil phase of the oil/water emulsion we used Solbrax Eco 225/255. This is a commercial solvent produced by Petrobras and was donated by its distribution subsidiary, BR Distribuidora. It is extracted from petroleum naphtha and composed mainly of aliphatic and naphthenic hydrocarbons with a distillation range between 498 and $528 \mathrm{~K}$. The solvent was characterized by carbon nuclear magnetic resonance (NMR-13C), by which Costa et al. [21] observed signals characteristic of linear or branched hydrocarbons and monocyclic alkanes. The presence of other compounds, such as alkenes, aromatics, or heteroatoms, was not observed.

2.3. Determination of the Phase Inversion Temperature (PIT) of the Systems. The PIT of the systems was determined with a Haake RS600 rheometer with a DG41 double-gap accessory, at a constant shear rate $(\dot{\gamma})$ of $30 \mathrm{~s}^{-1}$ and temperature range of $293-353 \mathrm{~K}$. The results were plotted on graphs of the logarithm of the viscosity $(\eta)$ in function of the temperature $(T)$. The dispersions were obtained by first adding the surfactant in the aqueous phase. Then after 24 hours the oil phase was added in the aqueous solution.

\subsection{Preparation and Characterization of the Oil/Water} Nanoemulsions by the PIT Method. According to Souza et al. [22], the process of preparing oil/water nanoemulsions by the PIT method consists of heating the system to temperatures near the PIT followed by rapid cooling, and the condition for nanoemulsion formation is the complete solubilization of the surfactant in the oil.

The Solbrax/water nanoemulsion was prepared by stirring the oil phase containing the surfactant (by a mechanical stirrer operating at $100 \mathrm{rpm}$ ) and then heating this phase and the aqueous phase separately to a temperature of $288 \mathrm{~K}$ above the corresponding PIT for each system. The purpose of the stirring and heating of the oil phase was to promote the complete solubilization of the surfactant. 
When the desired temperature was reached, the two phases were mixed, under stirring, and the mixture was immersed in bain-marie and cooled slowly until reaching 275 to $279 \mathrm{~K}$ below the predetermined PIT; at this point the system was cooled further to room temperature $(298 \mathrm{~K})$ in an ice bath.

2.5. Analysis of Size and Size Distribution of the Droplets of Prepared Emulsions. The emulsion so formed was then left under light stirring for $5 \mathrm{~min}$ and the size and size distribution of the droplets formed were analyzed in a Zetasizer Nano ZS particle size analyzer (Malvern) that works on the typical principal of dynamic light scattering. By the photon correlation spectroscopy method and the refraction index and viscosity values, we obtained quantitative results in the form of graphs of scattered light volume as a function of the particle size of each emulsion produced.

2.6. Use of the Nanoemulsions to Wash Sand Contaminated with Oil. The tests to study the removal of oil from the sand were performed in four steps: contamination of the sand, mixture of the contaminated sand with the nanoemulsion, extraction of the oil from the sand, and quantification of the oil removed from the sand. The sand was contaminated by adding $2 \mathrm{~g}$ of oil in $10 \mathrm{~g}$ of sand. After 24 hours, this mixture was agitated in a shaker bath at a moderate speed of 60 cycles $\cdot \min ^{-1}$ for 3 hours, at a temperature of $298 \mathrm{~K}$. Then the mixture was left to rest for 24 hours. (The crude oil used came from a Brazilian well and was donated by the Petrobras Research Center. It had the following characteristics: ${ }^{\circ} \mathrm{API}=$ 21.2; saturates content $=40.7 \%$; aromatics content $=34.1 \%$; resins content $=22.9 \%$; and asphaltenes content $=2.4 \%$. And the sand came from Mineração Jundu, with the following characteristics: grain size from 150 to 500 mesh, surface area $=0.2284 \mathrm{~m}^{2} / \mathrm{g}$, and pore diameter $=77.8430 \mathrm{~A}$.)

The cleaning was done by adding $30 \mathrm{~mL}$ of the nanoemulsion to this system and placing it in the shaker bath again. Another two flasks were filled with $30 \mathrm{~mL}$ of the nanoemulsion. One of these was left to rest at room temperature and the other was submitted to the same conditions as the flask containing the contaminated sand. The aim of this procedure was to observe the stability of the nanoemulsions submitted to the washing conditions.

To determine the best conditions to wash the contaminated sand, the time, temperature, and shaking speed in the shaker bath were varied. After the preestablished time, the flasks were removed from the shaker and left at rest for 24 hours, and then the nanoemulsion was separated out by centrifugation for characterization of the size of the dispersed droplets.

The process of extracting the oil from the sand was initially performed by adding two $30 \mathrm{~mL}$ aliquots of extractor solvent (Solbrax Eco 255/255 and toluene). This system was placed in the shaker bath at $298 \mathrm{~K}$ and agitated at $150 \mathrm{rpm}$ for 1 hour. Then the system was left at rest for approximately 1 hour and placed in a centrifuge at $3000 \mathrm{rpm}$ for $30 \mathrm{~min}$ to separate the sand from the solvent containing the oil. To guarantee that all of the oil was removed from the sand, the same procedure was repeated with another $30 \mathrm{~mL}$ aliquot of the extractor solvent.

The chemical makeup of the oil removed from the sand was evaluated in a Varian Cary 50 series ultravioletvisible spectrophotometer, using quartz cuvettes with a $2 \mathrm{~mm}$ optical pathway. The absorption intensity $(A)$ readings of the electromagnetic radiation at the wavelength utilized for the extraction solutions were obtained directly from the device, and the oil content was calculated with the help of its respective response curve plotted from solutions in the extractor solvent of known concentrations. The wavelength chosen for the measurements was $750 \mathrm{~nm}$, since at shorter wavelengths the absorption intensity values were off the device's scale or presented deviations from Lambert-Beer's Law [23].

\section{Results and Discussion}

3.1. Determination of the Cloud Point of the Nonionic Surfactants. The cloud point of aqueous solutions of nonionic surfactants is observed by increasing the temperature to the point where the ethylene oxide molecules become dehydrated and the surfactant separates out of the aqueous solution. The cloud point temperatures at concentrations of 10, 11, and $12 \mathrm{wt} \%$ are shown in Table 1.

It can be seen that an increasing surfactant concentration did not cause a significant variation in the cloud point values, which were within the measurement error range. The values presented in Table 1 are related to the first indication of clouding of the aqueous solutions of Ultrol L70. The clouding of the solution became more evident at higher temperatures (above $323 \mathrm{~K}$ ), and the complete separation was observed at temperatures near $333 \mathrm{~K}$.

3.2. Measurement of the Oil/Water Interfacial Tension. To produce stable nanoemulsions it is necessary for the oil/water interfacial tension to be minimized [24]. Therefore, analyses of this tension are very important, because they supply information about the surfactant concentrations at which sufficiently low interfacial tension values can be attained.

The interfacial tension of the water/Solbrax system was $19.5 \mathrm{mN} \cdot \mathrm{m}^{-1}$. Analysis of the oil/water interfacial tension values in Table 1 shows that the surfactant at the concentrations utilized reduced the tension values to very low levels.

\subsection{Determination of the PIT of the Systems by Rheometry.} The PIT values of the oil/water dispersions were determined by measuring the viscosity of the surfactant/water/Solbrax system at varied concentrations: $5 \mathrm{wt} \%$ Solbrax and 10 and $12 \mathrm{wt} \%$ surfactant (Figure 1) and $20 \mathrm{wt} \%$ Solbrax and 10 and $12 \mathrm{wt} \%$ surfactant (Figure 2).

The PITs of all the systems were above $338 \mathrm{~K}$, because Solbrax is mainly composed of aliphatic solvents, so the solubility inversion of the nonionic surfactant with increasing temperature is hindered, requiring higher temperatures. The lowest oil phase content $(5 \mathrm{wt} \%)$ also retarded the phase inversion of the system [14]. In the obtained graphs this 
TABLE 1: Cloud point of aqueous solutions of Ultrol L70 surfactant.

\begin{tabular}{lccc}
\hline Surfactant & Concentrations (wt.\%) & Cloud point $(\mathrm{K})$ & W/O interfacial tension $\left(\mathrm{mN} \cdot \mathrm{m}^{-1}\right)$ \\
\hline \multirow{3}{*}{ Ultrol L70 } & 10 & $306 \pm 1$ & $0.9 \pm 0.1$ \\
& 11 & $307 \pm 1$ & $0.5 \pm 0.1$ \\
& 12 & $308 \pm 1$ & $0.5 \pm 0.1$ \\
\hline
\end{tabular}

The analyses were made in triplicate and the results were presented as average of each measure with its standard deviation.

TABLE 2: Experimental matrix for each combination of levels of the washing process of the contaminated sand and their respective efficiencies.

\begin{tabular}{|c|c|c|c|c|c|c|c|}
\hline Systems & Time (h) & Speed (cycles. $\min ^{-1}$ ) & Temperature (K) & $X_{1}^{\mathrm{a}}$ & $X_{2}{ }^{\mathrm{a}}$ & $X_{3}{ }^{\mathrm{a}}$ & $E^{\mathrm{b}}(\%)$ \\
\hline 1 & 1 & 120 & 298 & -1 & -1 & -1 & 17.33 \\
\hline 2 & 1 & 120 & 318 & -1 & -1 & 1 & 39.13 \\
\hline 3 & 1 & 240 & 298 & -1 & 1 & -1 & 26.65 \\
\hline 4 & 1 & 240 & 318 & -1 & 1 & 1 & 68.03 \\
\hline 5 & 4 & 120 & 298 & 1 & -1 & -1 & 21.19 \\
\hline 6 & 4 & 120 & 318 & 1 & -1 & 1 & 83.29 \\
\hline 7 & 4 & 240 & 298 & 1 & 1 & -1 & 33.99 \\
\hline 8 & 4 & 240 & 318 & 1 & 1 & 1 & 95.78 \\
\hline 9 & 2.5 & 180 & 308 & 0 & 0 & 0 & 21.63 \\
\hline 10 & 2.5 & 180 & 308 & 0 & 0 & 0 & 22.20 \\
\hline 11 & 2.5 & 180 & 308 & 0 & 0 & 0 & 19.95 \\
\hline 12 & 2.5 & 180 & 308 & 0 & 0 & 0 & 19.44 \\
\hline 13 & 2.5 & 180 & 308 & 0 & 0 & 0 & 21.64 \\
\hline
\end{tabular}

${ }^{a}$ Representation of minimum, maximum and center points run. ${ }^{b}$ Efficiency calculated by the ratio of initial and final oil concentrations.

TABLE 3: Efficiency of the nanoemulsion of the washing process of the sand.

\begin{tabular}{|c|c|c|c|}
\hline \multicolumn{4}{|c|}{ Efficiency (\%) } \\
\hline Factors & Effect & Standard error & $P$ \\
\hline Average/interaction & 48.17 & 1.17 & 0.000 \\
\hline (1) Time (L) & 20.78 & 2.35 & 0.001 \\
\hline (2) Speed (L) & 15.88 & 2.35 & 0.001 \\
\hline (3) Temperature (L) & 46.77 & 2.35 & 0.001 \\
\hline 1 by 2 & -3.23 & 2.35 & 0.227 \\
\hline 1 by 3 & 15.18 & 2.35 & 0.001 \\
\hline 2 by 3 & 4.82 & 2.35 & 0.096 \\
\hline
\end{tabular}

transition was not well observed because the PIT is near the upper limit of the analysis.

3.4. Preparation and Characterization of the Nanoemulsions. The nanoemulsions obtained were characterized in the particle size analyzer. All the tests were run in triplicate. The graphs in Figures 3 and 4 show the average particle size distribution, accompanied by the respective error bars. The size of the droplets dispersed in the nanoemulsions was measured just after preparation (time 0 ) and at other time intervals until phase separation was observed.

It can be seen that as the concentration of the oil phase increased the size of the particles dispersed in the continuous phase increased as well. Also, the most stable nanoemulsion was that prepared with the lowest oil phase and the highest surfactant levels ( $5 \mathrm{wt} \%$ and $12 \mathrm{wt} \%$, resp., Figure $3(\mathrm{~b})$ ). This nanoemulsion was the only one still stable after four months.

3.5. Performance of the Nanoemulsions in Washing Sand Contaminated with Oil. There is growing interest in finding better strategies to clean up contaminated soil. In this study, we evaluated the use of nanoemulsions to remove crude oil from sand.

When sand particles are contaminated, the oil is retained both on the surface and in the pores of the grains. The physical-chemical process to clean the sand with emulsions entails dissolving the oil in the droplets dispersed in these systems. The main advantage of using nanoemulsions is that the droplets' small size allows them not only to dissolve the oil on the surface of the grains, but also to penetrate into the pores to remove the oil retained there as well.

We tested the nanoemulsion prepared with $12 \mathrm{wt} \%$ of the Ultrol L70 surfactant and $5 \mathrm{wt} \%$ of Solbrax. We chose this nanoemulsion, because it had the smallest particle size and the best stability among those tested previously.

3.6. Influence of Time, Shear Rate, and Temperature on the Efficiency of the Nanoemulsions to Wash Sand. We used a $2^{3}$ factorial experimental setup with center point run to analyze the performance of the nanoemulsion chosen in washing sand contaminated with oil. The parameters varied were temperature, shaking speed, and time in the shaker bath. The most stable nanoemulsion was formed by Solbrax as the oil phase was at surfactant concentrations of $5 \mathrm{wt} \%$ and $12 \mathrm{wt} \%$. 


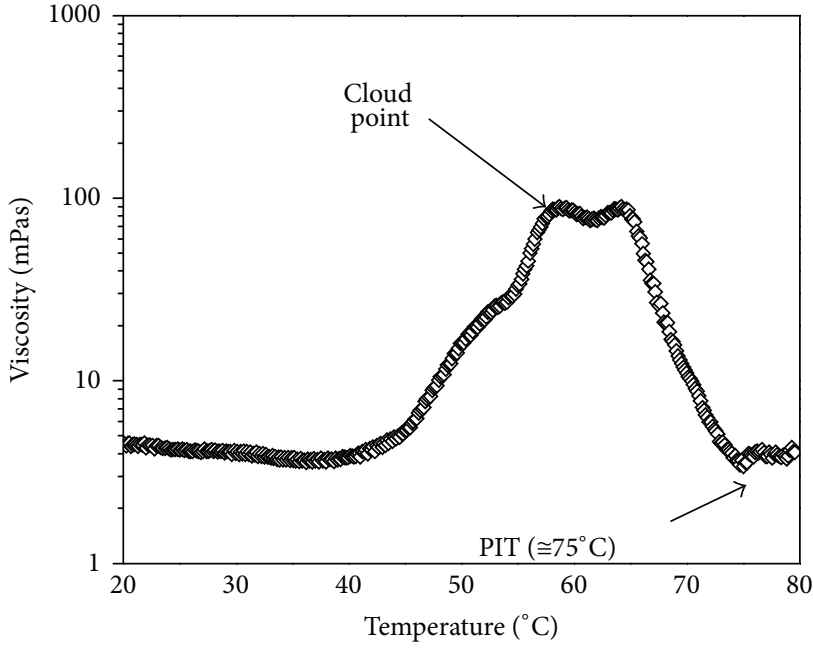

(a)

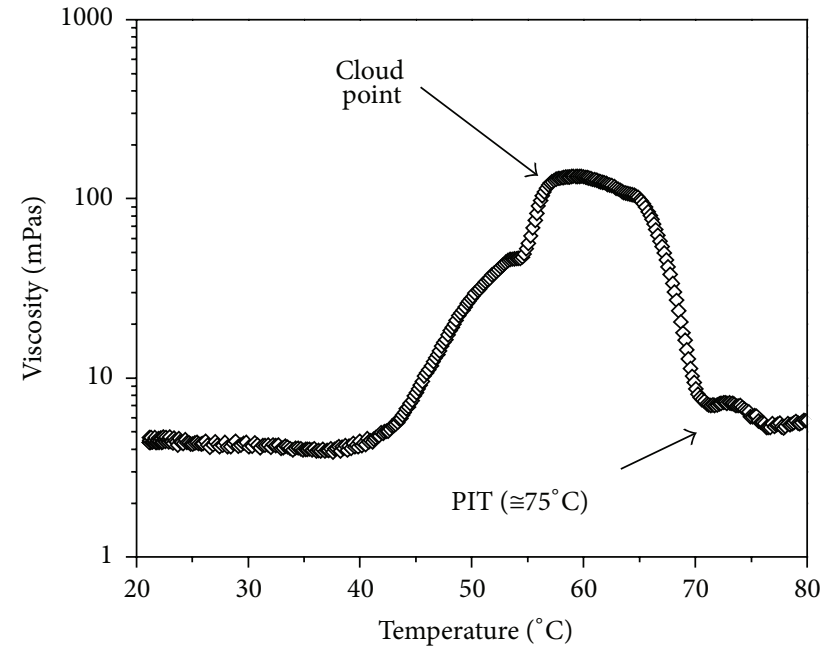

(b)

Figure 1: PIT for the systems of $5 \mathrm{wt} \%$ Solbrax and different concentrations of surfactant: (a) $10 \mathrm{wt} \%$ and (b) $12 \mathrm{wt} \%$.

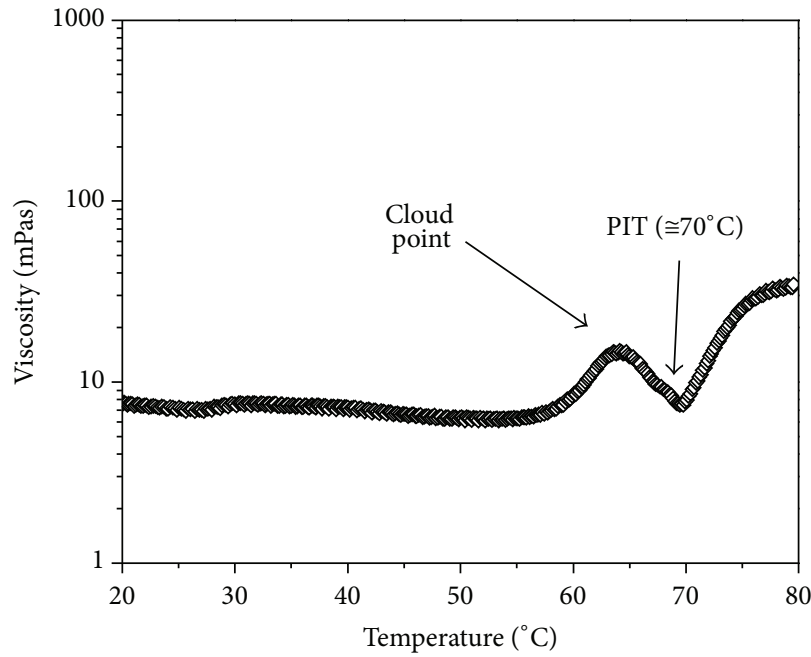

(a)

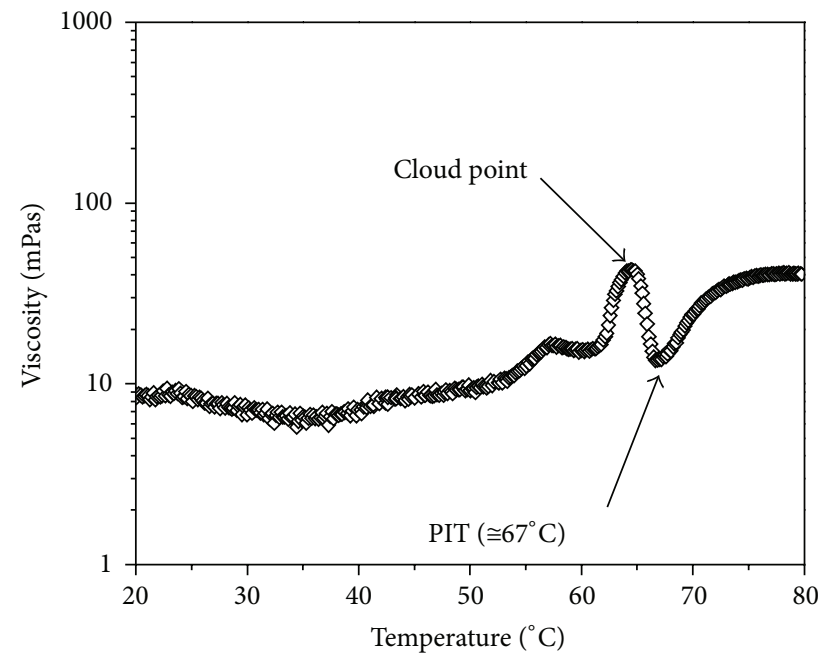

(b)

Figure 2: PIT for the systems of $20 \mathrm{wt} \%$ Solbrax and different concentrations of surfactant: (a) $10 \mathrm{wt} \%$ and (b) $12 \mathrm{wt} \%$.

From the results of the previous experiments, we set a level for each variable to determine the interval that would be analyzed. The starting point was a temperature of $308 \mathrm{~K}$, shear rate of 180 cycles $\mathrm{min}^{-1}$, and washing time of 2.5 hours (called the center point run: 0 ). The intervals used were $\pm 10^{\circ} \mathrm{C}, \pm 60$ cycles/min, and $\pm 1.5 \mathrm{~h}$. This design consists of 2 levels and 3 factors, requiring $2 \times 2 \times 2=8$ different tests. All told, we conducted 13 tests; 5 of them are repetitions at the center point run. Table 2 shows the experimental matrix and the efficiencies observed for each combination of levels.

We used the Statistica program to obtain the values of the effects of each parameter (time, speed, and temperature), as shown in Table 3, on the efficiency of the systems. In all cases, the results were considered significant when $P<0.05$.

As can be seen in Table 2, the nanoemulsion's efficiency in washing the oil-contaminated sand was significantly affected by the time and shear rate: the higher the factors, the more efficient the nanoemulsion. Regarding the interaction of one factor with the other, only the synergy between time and temperature was statistically significant. Figure 5 shows that the model proposed is able to represent the experimental data very well $\left(r^{2} \cong 1\right)$.

The results obtained show that the most efficient washing was attained at the highest temperature studied $(318 \mathrm{~K})$. The higher temperature probably facilitates the desorption process. Besides this, the washing time is more significant than the shear rate.

The predominance of these factors (temperature and time) can be explained by the low concentration of the oil phase in this emulsion (Solbrax Eco-5 wt\%). However, the system's good efficiency shows its potential application even at low Solbrax Eco levels. 


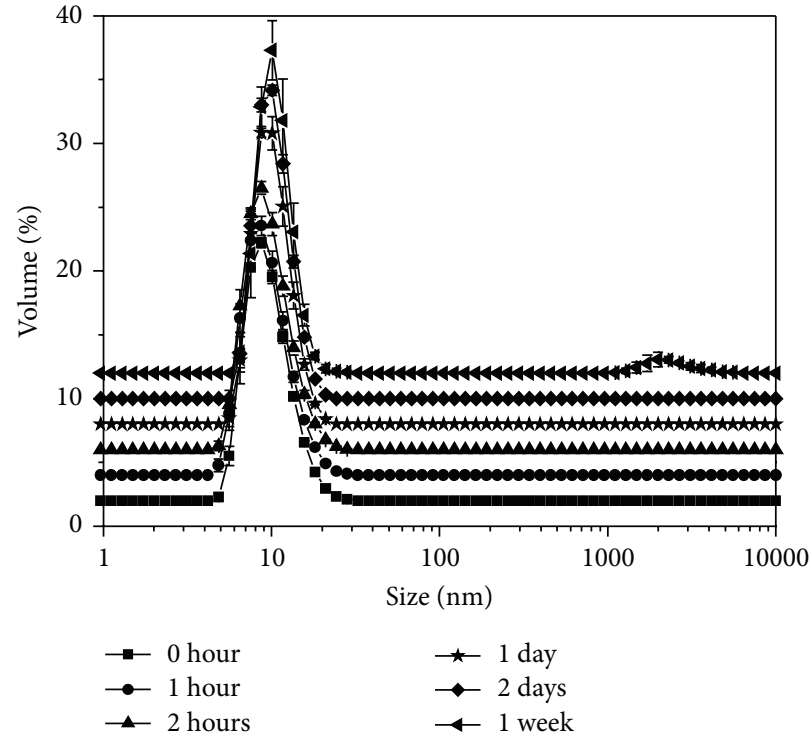

(a)

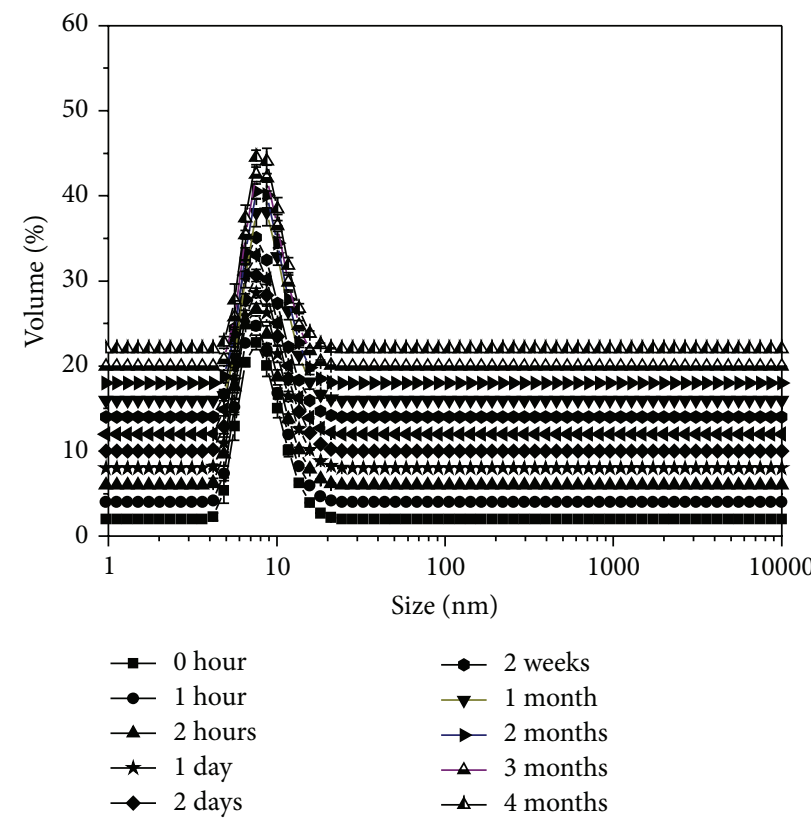

(b)

FIGURE 3: Particle size distribution of emulsions as a function of preparation time, using a $5 \mathrm{wt} \%$ Solbrax and different concentrations of surfactant: (a) $10 \mathrm{wt} \%$ and (b) $12 \mathrm{wt} \%$.

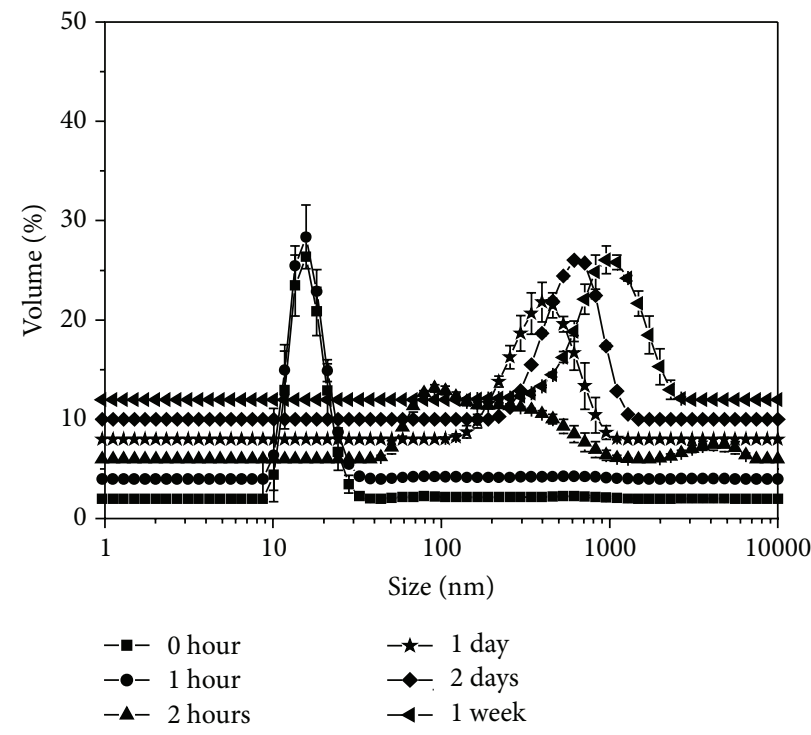

(a)

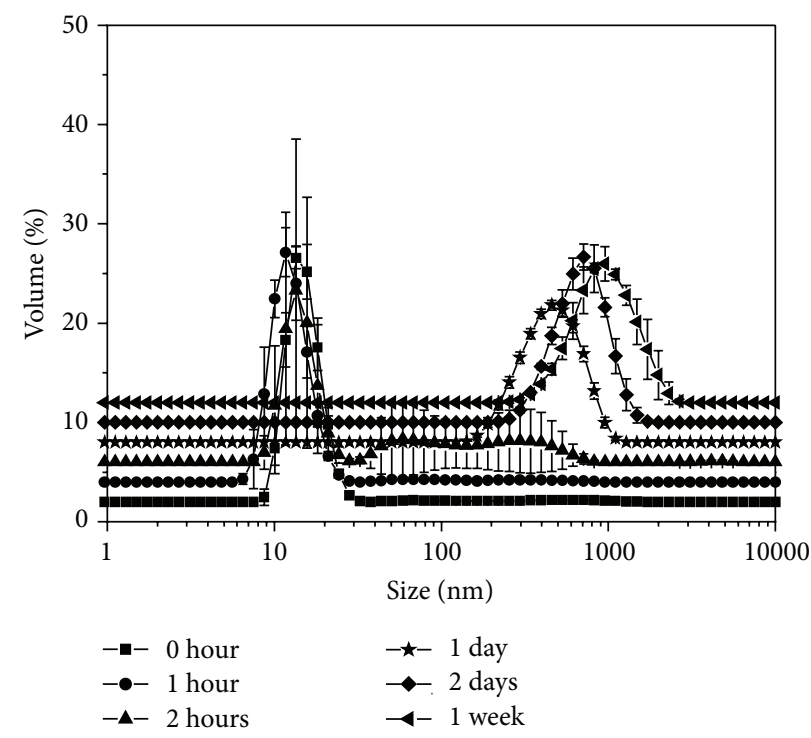

(b)

Figure 4: Particle size distribution of emulsions as a function of preparation time, using a 20 wt $\%$ Solbrax and different concentrations of surfactant: (a) $10 \mathrm{wt} \%$ and (b) $12 \mathrm{wt} \%$.

Finally, the nanoemulsions remained stable during the entire cleaning process, for extraction of the quantity of oil used in this work $(66,666 \mathrm{mg} / \mathrm{L})$. The nanoemulsion evaluated was stable with droplet sizes within the same range presented in Figure 3(b), even under the varied time and shear rate conditions to which it was submitted.
This behavior is desirable to ensure the nanoemulsion's efficiency in removing the contaminant in the oil phase. As the temperature was varied from 298 to $318 \mathrm{~K}$, there was a slight increase in the droplet size, which can be attributed to the lower solubility of the surfactant in the aqueous solution. 


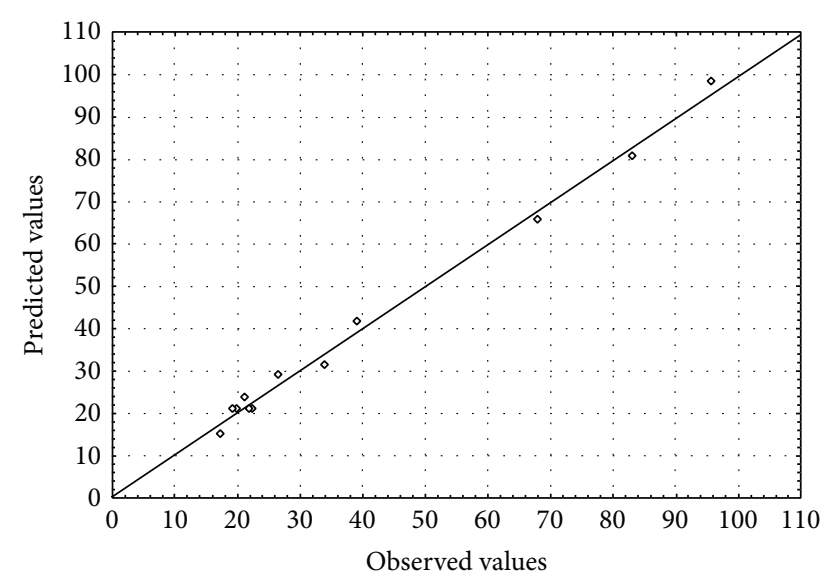

FiguRE 5: Observed versus predicted values of efficiency.

\section{Conclusions}

The phase inversion process caused by the variation in temperature (PIT), a known method to produce stable nanoemulsions, proved to be an effective method to produce stable nanoemulsions from Solbrax/water, in the presence of the nonionic ethoxylated lauryl ether surfactant (Ultrol L70), containing seven ethylene oxide units.

Efficiencies of around 90\% were achieved in removing oil from contaminated sand using the most stable nanoemulsion (containing 5 wt $\%$ oil phase and 12 wt\% Ultrol L70 surfactant).

The nanoemulsion utilized to wash the sand contaminated with crude oil remained stable, even when submitted to the conditions employed in this process (time, temperature, and shear rate).

\section{Conflict of Interests}

The authors declare that there is no conflict of interests regarding the publication of this paper.

\section{Acknowledgments}

The authors thank Oxiteno do Brasil for donating the polyoxide samples and the Petrobras Research Center (CENPES), the Coordinating Office for Improvement of University Researchers (CAPES), the National Council for Scientific and Technological Research (CNPq), the Office to Finance Studies and Projects (FINEP), and the National Petroleum Agency (ANP) for financial support.

\section{References}

[1] K. Urum, S. Grigson, T. Pekdemir, and S. McMenamy, "A comparison of the efficiency of different surfactants for removal of crude oil from contaminated soils," Chemosphere, vol. 62, no. 9, pp. 1403-1410, 2006.

[2] Q. Zhou, F. Sun, and R. Liu, "Joint chemical flushing of soils contaminated with petroleum hydrocarbons," Environment International, vol. 31, no. 6, pp. 835-839, 2005.
[3] L. L. Schramm, Surfactants: Fundamentals and Applications in the Petroleum Industry, Cambridge University Press, New York, NY, USA, 1st edition, 2000.

[4] D. H. Lee, R. D. Cody, D. J. Kim, and S. Choi, "Effect of soil texture on surfactant-based remediation of hydrophobic organic-contaminated soil," Environment International, vol. 27, no. 8, pp. 681-688, 2002.

[5] A. M. Al-Sabagh, N. S. Ahmed, A. M. Nassar, and M. M. Gabr, "Synthesis and evaluation of some polymeric surfactants for treating crude oil emulsions. Part I. Treatment of sandy soil polluted with crude oil by monomeric and polymeric surfactants," Colloids and Surfaces A: Physicochemical and Engineering Aspects, vol. 216, no. 1-3, pp. 9-19, 2003.

[6] M. S. Kuyukina, I. B. Ivshina, S. O. Makarov, L. V. Litvinenko, C. J. Cunningham, and J. C. Philp, "Effect of biosurfactants on crude oil desorption and mobilization in a soil system," Environment International, vol. 31, no. 2, pp. 155-161, 2005.

[7] K. Urum, T. Pekdemir, and M. Gopur, "Optimum conditions for washing of crude oil-contaminated soil with biosurfactant solutions," Process Safety and Environmental Protection, vol. 81, no. 3, pp. 203-209, 2003.

[8] K. Urum, T. Pekdemir, D. Ross, and S. Grigson, "Crude oil contaminated soil washing in air sparging assisted stirred tank reactor using biosurfactants," Chemosphere, vol. 60, no. 3, pp. 334-343, 2005

[9] M. C. K. Oliveira and G. G. Maldonado, PI 0605007-7. Composição para remediação de solos e resíduos sólidos contaminados por hidrocarbonetos de alto peso molecular, 2006.

[10] C. L. Yap, S. Gan, and H. K. Ng, "Application of vegetable oils in the treatment of polycyclic aromatic hydrocarbonscontaminated soils," Journal of Hazardous Materials, vol. 177, no. 1-3, pp. 28-41, 2010.

[11] P. F. Oliveira, L. S. Spinelli, and C. R. E. Mansur, "The application of nanoemulsions with different orange oil concentrations to remediate petroleum-contaminated soil," Journal of Nanoscience and Nanotechnology, vol. 12, no. 5, pp. 4081-4087, 2012.

[12] T. Tadros, P. Izquierdo, J. Esquena, and C. Solans, "Formation and stability of nano-emulsions," Advances in Colloid and Interface Science, vol. 108-109, pp. 303-318, 2004.

[13] K. Ouzineb, C. Lord, N. Lesauze, C. Graillat, P. A. Tanguy, and T. McKenna, "Homogenisation devices for the production of miniemulsions," Chemical Engineering Science, vol. 61, no. 9, pp. 2994-3000, 2006.

[14] V. B. Souza, L. S. Spinelli, G. Gonzalez, and C. R. E. Mansur, "Determination of the phase inversion temperature of orange oil/water emulsions by rheology and microcalorimetry," Analytical Letters, vol. 42, no. 17, pp. 2864-2878, 2009.

[15] L. S. Spinelli, C. R. E. Mansur, G. González, and E. F. Lucas, "Evaluation of process conditions and characterization of particle size and stability of oil-in-water nanoemulsions," Colloid Journal, vol. 72, no. 1, pp. 56-65, 2010.

[16] L. R. Kourniatis, L. S. Spinelli, G. González, and C. R. E. Mansur, "Nanoemulsões óleo de laranja/água preparadas em homogeneizador de alta pressão," Química Nova, vol. 33, pp. 295-300, 2010.

[17] L. R. Kourniatis, L. S. Spinelli, C. R. Piombini, and C. R. E. Mansur, "Formation of orange oil-in-water nanoemullsions using nonionic surfactant mixtures by high pressure homogenizer," Colloid Journal, vol. 72, no. 3, pp. 396-402, 2010. 
[18] D. J. Miller, T. Henning, and W. Grünbein, "Phase inversion of W/O emulsions by adding hydrophilic surfactant-a technique for making cosmetics products," Colloids and Surfaces A: Physicochemical and Engineering Aspects, vol. 183-185, pp. 681688, 2001.

[19] E. W. Duck, "Emulsion polymerization," in Encyclopedia of Polymer Science and Technology, H. F. Mark, N. G. Gaylord, and N. M. Bikales, Eds., vol. 5, pp. 801-859, Wiley-Interscience, New York, NY, USA, 1966.

[20] N. Anton, P. Gayet, J.-P. Benoit, and P. Saulnier, "Nanoemulsions and nanocapsules by the PIT method: an investigation on the role of the temperature cycling on the emulsion phase inversion," International Journal of Pharmaceutics, vol. 344, no. 1-2, pp. 44-52, 2007.

[21] J. A. Costa, E. F. Lucas, Y. G. C. Queirós, and C. R. E. Mansur, "Evaluation of nanoemulsions in the cleaning of polymeric resins," Colloids and Surfaces A: Physicochemical and Engineering Aspects, vol. 415, pp. 112-118, 2012.

[22] V. B. Souza, S. M. Almeida, L. S. Spinelli, and C. R. E. Mansur, "Stability of orange oil/water nanoemulsions prepared by the PIT method," Journal of Nanoscience and Nanotechnology, vol. 11, no. 3, pp. 2237-2243, 2011.

[23] J. A. McDonald and A. R. Rennie, "A structural study of mixed micelles containing C16TAB and C12E6 surfactants," Langmuir, vol. 11, no. 5, pp. 1493-1499, 1995.

[24] D. J. Shaw, Introdução à Química dos Colóides e de Superfícies, Edgard Blücher Ltda, São Paulo, Brazil, 1975. 

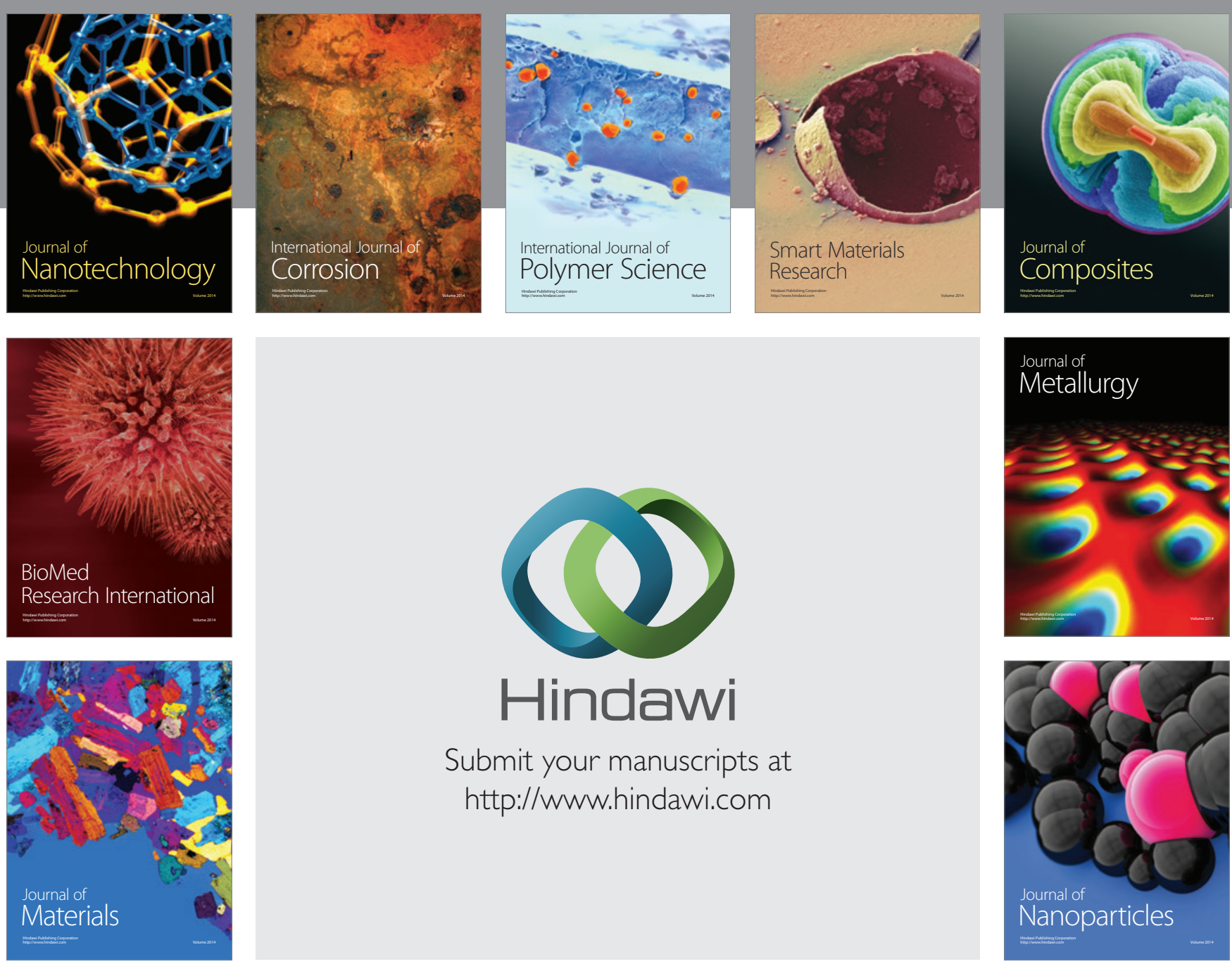

Submit your manuscripts at http://www.hindawi.com
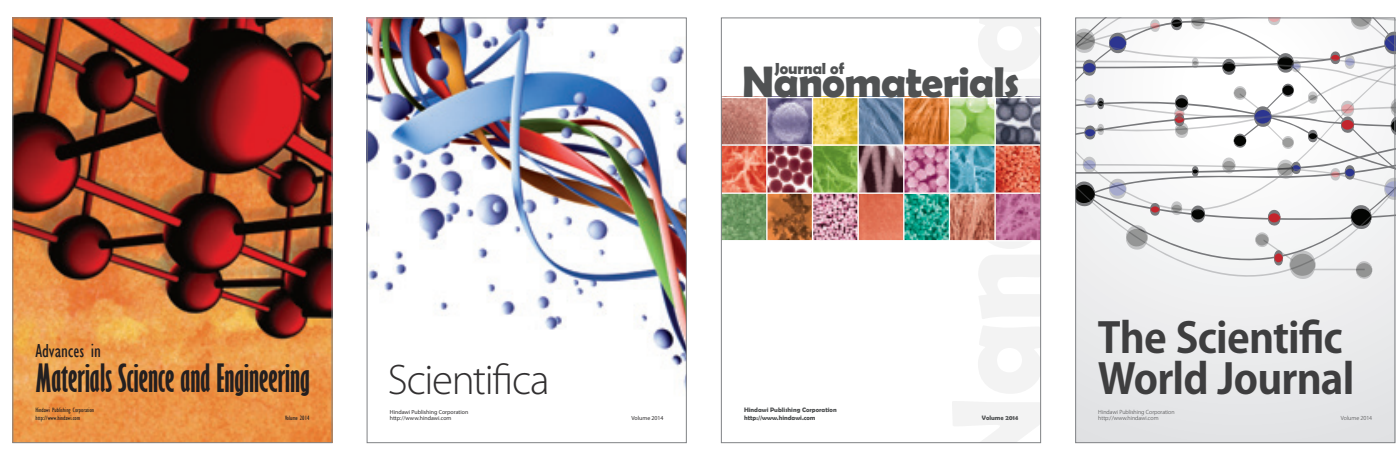

\section{The Scientific World Journal}
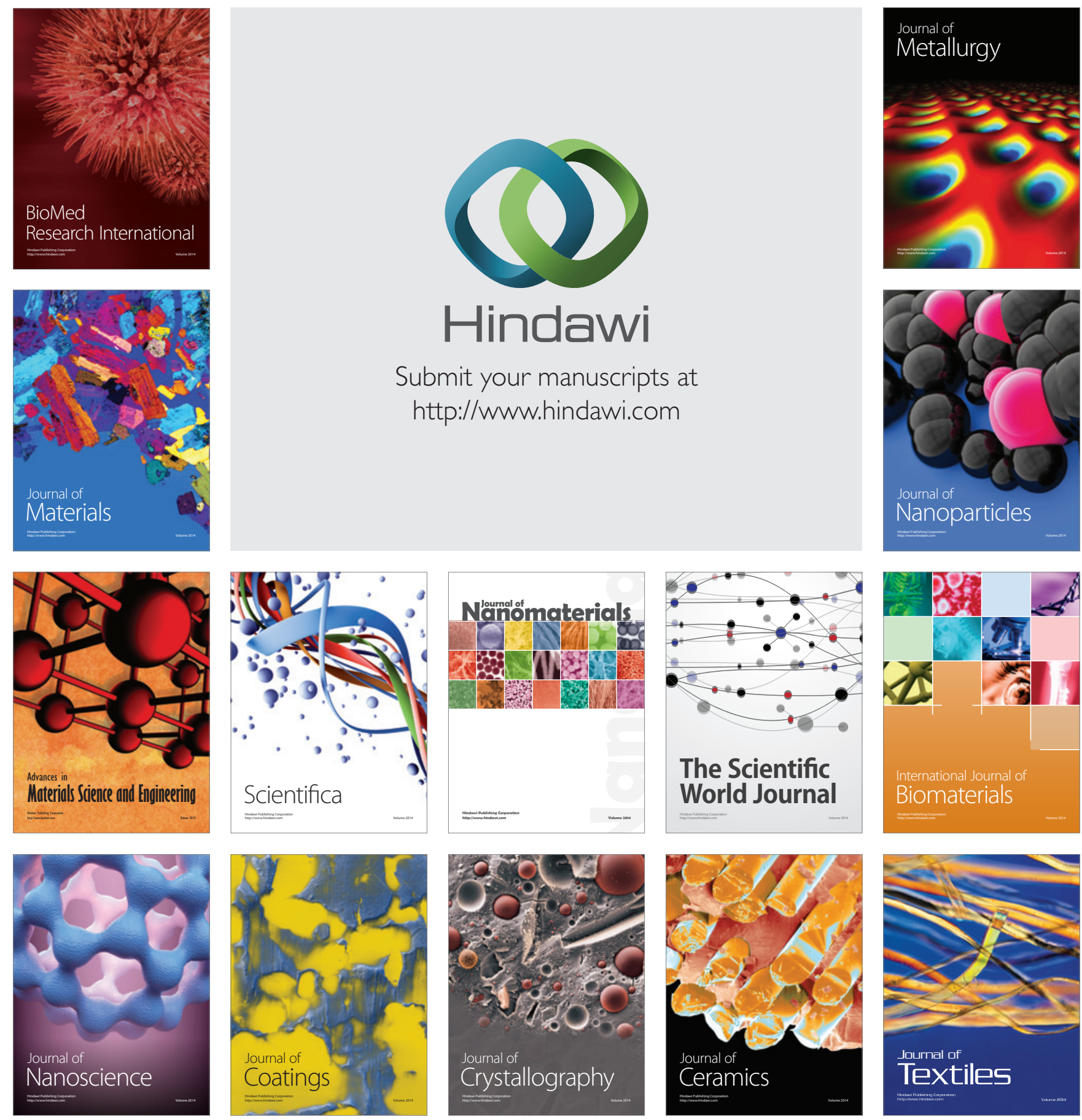